\title{
In vitro fermentability of dextran, oligodextran and maltodextrin by human gut bacteria
}

\author{
Estibaliz Olano-Martin, Konstantinos C. Mountzouris, Glenn R. Gibson and Robert A. Rastall* \\ Department of Food Science and Technology, The University of Reading, PO Box 226, Whiteknights, Reading RG6 6AP, UK
}

(Received 14 January 1999 - Revised 9 September 1999 - Accepted 27 September 1999)

\begin{abstract}
Anaerobic batch culture fermenters were used for a preliminary screening of the in vitro utilization by human gut microflora of dextran and novel oligodextrans (I, II and III) produced in the University of Reading (UK). Glucose and fructooligosaccharides (FOS) were used as reference carbohydrates. As expected, FOS acted as a good prebiotic in that it selectively increased numbers of bifidobacteria in the early stages of the fermentation. Dextran and oligodextrans each resulted in an enrichment of bifidobacteria in the batch cultures, with high levels of persistence up to $48 \mathrm{~h}$. They also produced elevated levels of butyrate ranging from 5 to $14.85 \mathrm{mmol} / \mathrm{l}$. To more effectively simulate conditions that prevail in different regions of the large intestine, a three-stage continuous culture cascade system was used to study further the fermentation of dextran, a low-molecular-mass oligodextran (IV) and maltodextrin. Oligodextran IV was shown to be the best substrate for bifidobacteria and lactobacilli with steady-state populations of bifidobacteria and lactobacilli being higher in all three vessels of the gut model than the respective populations resulting from dextran and maltodextrin. A maximum difference of $1.9 \mathrm{log}$ was observed in vessel 1 for both bifidobacteria and lactobacilli in the case of dextran fermentation, while $1.4 \mathrm{log}$ and $0.8 \mathrm{log}$ in vessel 3 were the maximum differences for bifidobacteria and lactobacilli when maltodextrin was used as the carbohydrate source. Moreover, dextran and oligodextran appeared to stimulate butyrate production, with a maximum production up to $25.39 \mathrm{mmol} / \mathrm{l}$ in vessel 3 when fermenting dextran, followed by $21.70 \mathrm{mmol} / \mathrm{l}$ in the case of oligodextran IV and only $12.64 \mathrm{mmol} / \mathrm{l}$ in the case of maltodextrin.
\end{abstract}

Oligosaccharides: Gut fermentation: Prebiotics: Butyrate

The microflora present in the human large intestine is thought to comprise at least 400 culturable bacterial species, although the likely microbial diversity is much greater than this (Gibson \& Roberfroid, 1995). As a result of the metabolic activities of different bacteria, this complex ecological niche plays a major role in human nutrition, and possibly health (McCartney \& Gibson, 1998).

Many undigested food materials, principally carbohydrates and proteins, can enter the large intestine and are susceptible to metabolism by the microbiota. In general, gut fermentation is characterized by carbohydrate metabolism in the proximal colon, with increased proteolysis in the distal gut (Macfarlane et al. 1992). Short-chain fatty acids (SCFA), particularly butyrate, and vitamins are desirable end-products of this process. Physiologically undesirable metabolites include carcinogens, other tumour-promoters and bacterial toxins (Cummings \& Macfarlane, 1991; Tomomatsu, 1994). Because of this dichotomy of activities, there is currently much interest in determining appropriate dietary components that stimulate a benign, or beneficial, gut microflora.

Despite our relative lack of understanding of the ecological interactions between bacterial species in the colon, it is possible to identify certain markers that may be associated with a healthy hindgut. These include populations of species belonging to the genera Lactobacillus and Bifidobacterium (Mizota, 1996; Yaeshima, 1996) and the production of butyrate (Cummings, 1984; Cummings \& Macfarlane, 1991). Butyrate acts as the preferred energy source for colonic epithelial cells (Delzenne \& Roberfroid, 1994) and it has been shown to stimulate apoptosis in colon cells in vitro (Hague et al. 1995). Lactobacilli and bifidobacteria may inhibit gut colonization by pathogens and are thought to exert other protective effects against acute and chronic gut disorders (Fuller, 1989). For this reason, current prebiotics are largely targeted towards these genera. A prebiotic has been defined by Gibson \& Roberfroid (1995) as 'a non-digestible food ingredient that beneficially affects

\footnotetext{
Abbreviations: DP, degree of polymerization; FOS, fructooligosaccharides; IMO, isomaltooligosaccharides; LAB, lactic acid bacteria; SCFA, short-chain fatty acids; V, vessel.

* Corresponding author: Dr Robert A. Rastall, fax +44 (0) 118931 0080, email r.a.rastall@afnovell.reading.ac.uk
} 
the host by selectively stimulating the growth and/or activity of one or a limited number of bacteria in the colon, and thus improves host health'.

Currently a commercially produced isomaltooligosaccharide (IMO) preparation is the market leader in the prebiotic oligosaccharide sector in Japan (Japanscan, 1998), with annual sales of approximately 10000 tonnes. This commercial preparation is a mixture of IMO such as isomaltose $(230 \mathrm{~g} / \mathrm{kg})$ and isomaltotriose $(170 \mathrm{~g} / \mathrm{kg})$ but it also contains high amounts (approximately $300 \mathrm{~g} / \mathrm{kg}$ ) of other non-isomalto oligosaccharides such as panose, maltose, maltotriose, nigerose and kojibiose. Approximately $260 \mathrm{~g} / \mathrm{kg}$ of this preparation consists of higher oligosaccharides having a degree of polymerization (DP) ranging from 4 to 6. IMO are partly digestible in the small intestine, while the degree of digestion decreases for components having DP $\geqslant 3$ (Kaneko et al. 1995).

The development of a prebiotic that allows the growth of elevated numbers of lactic acid bacteria (LAB), whilst stimulating butyrate production, would be advantageous. We have recently investigated ways of generating oligodextrans with controlled physico-chemical characteristics using enzyme biotechnology. The manufacturing process involves the enzymic depolymerization of industrial-grade dextran using an endo-dextranase in membrane bioreactors (Mountzouris et al. 1999) to effectively control the molecular size of the oligodextran products. It is expected that dextran and the produced oligodextrans would be less digestible than IMO for two main reasons: (a) their predominant $\alpha-1 \rightarrow 6$ glucosidic linkages would escape digestion by the luminal enzymes, and (b) their higher molecular mass components (i.e. $\mathrm{DP} \geqslant 3$ ) would be also less susceptible to membrane digestion by the disaccharidases on the epithelial surface of the small intestine. Thus, the oligodextrans developed as described earlier would be expected to reach the colon more-or-less intact as they were eaten.

The aim of the present work was to generate some information on the in vitro fermentability of oligodextrans by human gut microflora. In vitro fermentations using batch and continuous culture methods have been regarded as acceptable models for the study of large intestinal function
(Salminen et al. 1998). Initially, batch culture fermentations were considered as the only possible option for experimentation due to the limited amounts of the novel oligodextrans produced on a laboratory scale according to the method of Mountzouris et al. (1999). The study of the in vitro fermentability of one oligodextran type in a more efficient validated three-stage continuous culture system (Macfarlane et al. 1998) was enabled, following the development of a continuous membrane bioreactor system (KC Mountzouris, unpublished results) facilitating the production of oligodextrans in larger amounts. Dextran and a food-grade maltodextrin were also tested using the three-stage continuous culture system (Macfarlane et al. 1998), the former because it is the polysaccharide used in the production of the oligodextrans and the latter because starch is associated with high in vitro production of butyrate (Wang \& Gibson, 1993).

\section{Materials and methods \\ General reagents}

Unless otherwise specified, all chemicals were purchased from Sigma (Poole, Dorset, UK). Microbiological culture media and other additions were obtained from Oxoid (Basingstoke, Hants., UK). Fructooligosaccharides (FOS; Oligofructose P95) were obtained from Orafti (Tienen, Belgium). A food-grade maltodextrin preparation, 'Goldex 20 ' with a dextrose equivalent value in the range of 17-20, was kindly supplied by ABR Foods Ltd (Corby, Northants., UK).

The oligodextrans are currently manufactured according to a process developed by Mountzouris et al. (1999). Three different sizes of oligodextrans were used in the batch culture experiment: oligodextrans I, II, and III. Another oligodextran (IV) produced in a continuous process $(\mathrm{KC}$ Mountzouris, unpublished results) was used for the gut model. The composition of the oligodextrans with respect to their DP is given in Table 1 and the overall molecular mass distributions of oligodextrans I, II and III are shown in Fig. 1.

Table 1. Composition $(\mathrm{g} / \mathrm{kg})$ of oligodextrans used in the batch and gut model experiments with respect to their degree of glucose polymerization (DP)

\begin{tabular}{|c|c|c|c|c|}
\hline & $\begin{array}{l}\text { Oligodextran I* } \\
(\mathrm{g} / \mathrm{kg})\end{array}$ & $\begin{array}{l}\text { Oligodextran II* } \\
(\mathrm{g} / \mathrm{kg})\end{array}$ & $\begin{array}{c}\text { Oligodextran III* } \\
(\mathrm{g} / \mathrm{kg})\end{array}$ & $\begin{array}{c}\text { Oligodextran IV } † \\
(\mathrm{~g} / \mathrm{kg})\end{array}$ \\
\hline$\overline{\mathrm{DP} 1}$ & 62.2 & 75.2 & 151.3 & 9.8 \\
\hline DP 2 & 185.3 & $50 \cdot 7$ & 0.0 & 154.6 \\
\hline DP 3 & 269.6 & $98 \cdot 2$ & $58 \cdot 2$ & 264.6 \\
\hline DP 4 & $41 \cdot 1$ & 27.9 & 11.6 & $46 \cdot 1$ \\
\hline DP 5 & 11.4 & $12 \cdot 4$ & $6 \cdot 6$ & 14.8 \\
\hline DP 6 & 14.3 & 12.9 & 8.3 & $22 \cdot 1$ \\
\hline DP 7 & 20.0 & 13.5 & 8.8 & 31.8 \\
\hline DP 8 & $22 \cdot 2$ & 14.7 & $8 \cdot 3$ & $36 \cdot 6$ \\
\hline DP 9 & 18.4 & 14.5 & $7 \cdot 6$ & $32 \cdot 8$ \\
\hline DP 10 & 12.9 & $15 \cdot 0$ & 7.6 & 28.4 \\
\hline DP $11+$ & 342.4 & 665.0 & $731 \cdot 7$ & 358.5 \\
\hline
\end{tabular}

\footnotetext{
* Oligodextrans used in the batch experiments. The molecular mass distributions of oligodextran I, II and III are shown in Fig. 1.

†Oligodextran used in the three-stage continuous culture experiment.
} 


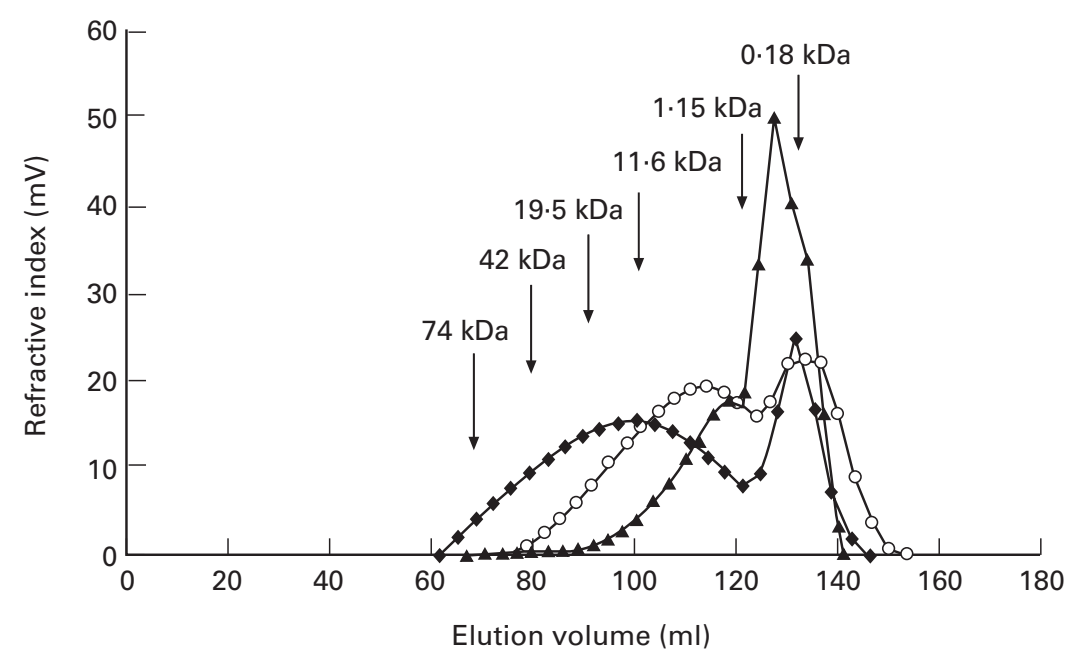

Fig. 1. Molecular mass distributions of oligodextran I ( $\mathbf{\Delta})$, oligodextran II (O) and oligodextran III ( ) used in the batch culture fermentations. Arrows indicate the elution volumes of the standards used to calibrate the chromatographic column (Sephacryl S-200 HR). Data were adapted from Mountzouris et al. (1999).

\section{Batch culture fermentations}

Sterile batch culture fermenters ( $50 \mathrm{ml}$ capacity) were filled with a pre-reduced culture medium and inoculated with slurried faecal bacteria to give a final concentration of $100 \mathrm{~g} / \mathrm{l}$. Carbohydrates (glucose, oligodextran I, oligodextran II, oligodextran III, dextran, FOS) were added separately before inoculation, to give a final concentration of $10 \mathrm{~g} / \mathrm{l}$. The closed fermenters were maintained under a headspace of $\mathrm{O}_{2}$-free $\mathrm{N}_{2}$. Faecal slurries were prepared by homogenizing freshly voided faeces in anoxic $0 \cdot 1 \mathrm{M}$-sodium phosphate buffer, $\mathrm{pH} 7 \cdot 0$. Details of the process are given by Wang \& Gibson (1993).

The basal culture medium consisted of $(\mathrm{g} / \mathrm{l})$ : peptone water $2 \cdot 0$, yeast extract $2 \cdot 0, \mathrm{NaCl} 0 \cdot 1, \mathrm{~K}_{2} \mathrm{HPO}_{4} 0 \cdot 04$,

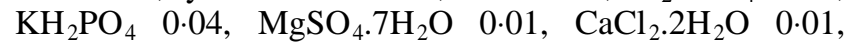
$\mathrm{NaHCO}_{3} 2 \cdot 0$, cysteine $\mathrm{HCl} 0 \cdot 5$, bile salts $0 \cdot 5$. The following liquid additions were made $(\mathrm{ml} / \mathrm{l})$ : Tween 802 , phylloquinone 10; haemin solution 1 .

Fermenters were incubated at $37^{\circ}$ and triplicate samples ( $1 \mathrm{ml}$ ) removed after $0,6,24$ and $48 \mathrm{~h}$ for the enumeration of bacteria on appropriate agars and analysis of fermentation end-products. The initial $\mathrm{pH}$ of the culture medium was $7 \cdot 0$ and was not controlled during the incubation period. All additions, inoculations and incubation, sampling and plating were carried out inside an anaerobic cabinet containing $\mathrm{H}_{2}-$ $\mathrm{CO}_{2}-\mathrm{N}_{2}$ (10:10:80, by vol.).

\section{Three-stage continuous culture system}

To simulate the different physical and nutritional characteristics of the proximal, transverse and distal colons, a threestage continuous culture (gut model) system was inoculated with a faecal bacterial inoculum. The system comprised a cascade of three fermenters, connected in series, such that culture medium was pumped into vessel (V) 1, which sequentially fed V2 then V3 and ultimately a waste unit. V1 and V2 had operating volumes of $250 \mathrm{ml}$, whilst the volume in V3 was $300 \mathrm{ml}$. The respective $\mathrm{pH}$ values in each of the fermenters were $5 \cdot 5,6 \cdot 2$ and $7 \cdot 1$. Each vessel was magnetically stirred and maintained under anaerobic conditions by continuous sparging with $\mathrm{O}_{2}$-free $\mathrm{N}_{2}$. Temperature $\left(37^{\circ}\right)$ and $\mathrm{pH}$ were controlled automatically. As such V1 simulated the acidic conditions, fast bacterial growth and high substrate availability that prevail in the proximal (right) colon. In contrast, V3 was held at a neutral $\mathrm{pH}$, bacteria grew more slowly and were substrate limited, conditions similar to those in the distal (left) colon. Overall retention time in the system was $60 \mathrm{~h}$. The system has been previously validated as giving a close approximation to fermentation events in situ (Macfarlane et al. 1998). Culture media and faecal slurries were prepared as previously described.

One experiment was carried out for each $\mathrm{C}$ source: dextran, oligodextran IV and maltodextrin. Dilution rates in the system were $0 \cdot 016 / \mathrm{h}$ for dextran and maltodextrin and $0 \cdot 02 / \mathrm{h}$ for oligodextran IV. The carbohydrate source was added to the fermentation medium before autoclaving to give a final concentration of $10 \mathrm{~g} / \mathrm{l}$. The vessels were filled with $200 \mathrm{ml}$ fermentation medium and $50 \mathrm{ml}$ inoculum.

The system was allowed to equilibrate for $24 \mathrm{~h}$, after which time the medium pump was started. Samples $(1 \mathrm{ml})$ were removed from the inoculum after at least seven turnovers from each vessel for the enumeration of selected bacterial genera and fermentation end-product analyses. Sampling from each vessel was performed in triplicate.

\section{Enumeration of bacteria}

Samples $(1 \mathrm{ml})$ of batch or continuous culture were serially diluted to $10^{-7}$ in an anaerobic cabinet with pre-reduced half strength peptone water, enriched with $0.5 \mathrm{~g}$ cysteine $\mathrm{HCl} / \mathrm{l}$. Portions $(20 \mu \mathrm{l})$ of each dilution were then inoculated onto a range of agars designed to be selective for predominant colonic bacteria. The following growth media were used: 
nutrient agar (total aerobes), Wilkins Chalgren agar (total anaerobes), MacConkey agar no. 3 (coliforms), Rogosa agar (lactobacilli), reinforced clostridia agar (clostridia), Beeren's agar (bifidobacteria), Brucella agar (bacteroides). Various additions were made to the agars in order to improve selectivity (Wang \& Gibson, 1993). Agar plates were placed into an anaerobic cabinet at least $16 \mathrm{~h}$ before inoculation to allow them to pre-reduce. After incubation, single colonies were counted and characterized to genus level on the basis of colonial appearance, Gram reaction, microscopic characteristics and limited biochemical profiles as described by Wang \& Gibson (1993). For further confirmation of culture identity, some randomly selected isolates were further characterized by sequencing specific regions of 16S-rRNA (McCartney \& Gibson, 1998).

\section{Analysis of fermentation end-products}

Samples $(1 \mathrm{ml})$ from the cultures were centrifuged at $13000 \mathrm{~g}$ for $15 \mathrm{~min}$ to remove bacteria and other particulate material.

Analysis of SCFA was carried out by HPLC. Acetate, propionate and butyrate were determined on a BIO-RAD Aminex HPX-87H column $(300 \times 7.8 \mathrm{~mm})$. Degassed $0.005 \mathrm{M}-\mathrm{H}_{2} \mathrm{SO}_{4}$ was used as eluent at a flow rate of $0.6 \mathrm{ml} / \mathrm{min}$ and an operating temperature of $50^{\circ}$. Organic acids were detected by u.v. absorbance at a wavelength of $215 \mathrm{~nm}$. The injection volume was $20 \mu \mathrm{l}$ sample.

Residual carbohydrate in the samples was determined by the phenol-sulfuric assay for total sugar (Dubois et al. 1956).

\section{Results}

\section{Influence of molecular mass on the fermentation of oligodextrans in batch culture}

Counts of selected bacterial genera are shown in Fig. 2. During the first $6 \mathrm{~h}$ of fermentation, counts of total anaerobes were seen to increase slightly giving a maximum increase of $0.5 \log$ in the case of dextran. Generally, high levels of total anaerobes were maintained in the batch systems growing on oligodextrans, dextran and FOS for up to $48 \mathrm{~h}$, the effect being more pronounced in the case of oligodextrans II and III and dextran. The oligodextrans II, III and dextran were shown to allow clostridia counts (Fig. 2) to persist for up to $24 \mathrm{~h}$, but this did not happen in the case of FOS and oligodextran I.

Oligodextran I and FOS (Fig. 2) displayed quite similar behaviour with respect to their selectivity towards Bacteroides and bifidobacteria. A decrease in Bacteroides counts by 1.6 and $2.3 \log$ occurred during the first $6 \mathrm{~h}$ for oligodextran I and FOS respectively. Bifidobacteria increased by a maximum of $0.6 \log$ within the first $6 \mathrm{~h}$ in the case of oligodextran I, and by $0.4 \mathrm{log}$ in the case of FOS. High levels of bifidobacteria appeared to persist for up to $48 \mathrm{~h}$ mostly in the case of oligodextrans II, III and dextran.

Generation of SCFA (Fig. 3) was used to determine the fermentability of the different carbohydrate substrates in the batch culture fermenters. All the carbohydrates tested were utilized to some degree (Fig. 4) but SCFA production patterns were variable (Fig. 3).

The utilization of the oligodextrans showed dependence
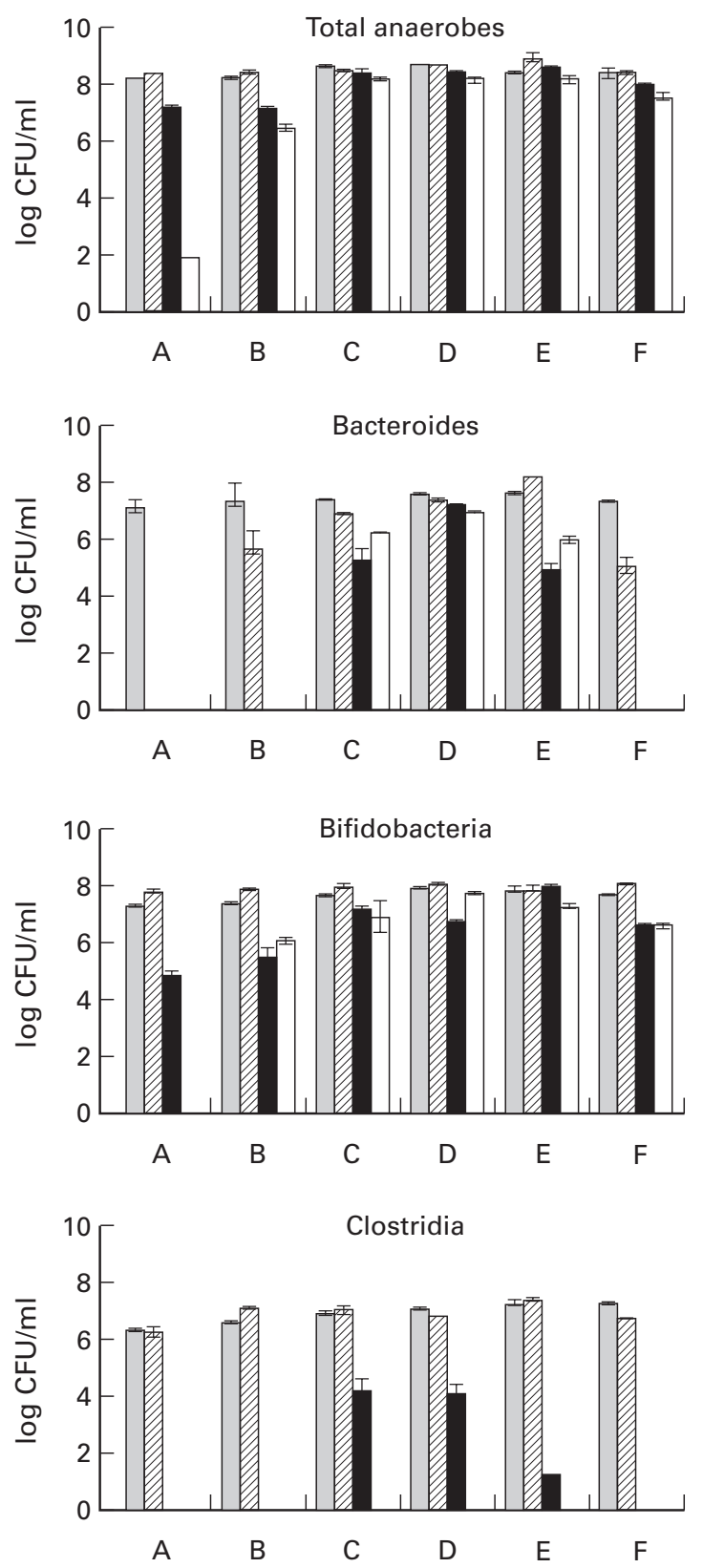

Fig. 2. Counts (log colony-forming units $(\mathrm{CFU}) / \mathrm{ml}$ ) of selected gut

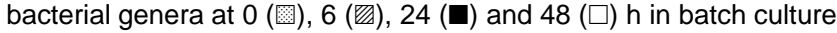
experiments using glucose $(A)$, oligodextran I (B), oligodextran II (C), oligodextran III (D), dextran (E) and fructooligosaccharides (F) as carbohydrate sources. Values are means of triplicate determinations on the same culture, with standard deviations represented by vertical bars.

on their molecular mass: glucose $>$ oligodextran I $>$ oligodextran II $>$ oligodextran III $>$ dextran. FOS had its own utilization pattern (up to $50 \%$ within $48 \mathrm{~h}$ ) which ranged between those of oligodextrans I and III (Fig. 4).

The SCFA data (Fig. 3) showed that carbohydrates with predominantly $\alpha-1 \rightarrow 6$ glucosidic linkages (dextran and oligodextrans) were good substrates for butyrate formation. Both the low-molecular-mass oligodextran I and FOS had similar SCFA patterns (Fig. 3). Their fermentation did not produce large quantities of butyrate; $3.9 \mathrm{mmol} / \mathrm{l}$ for FOS and 

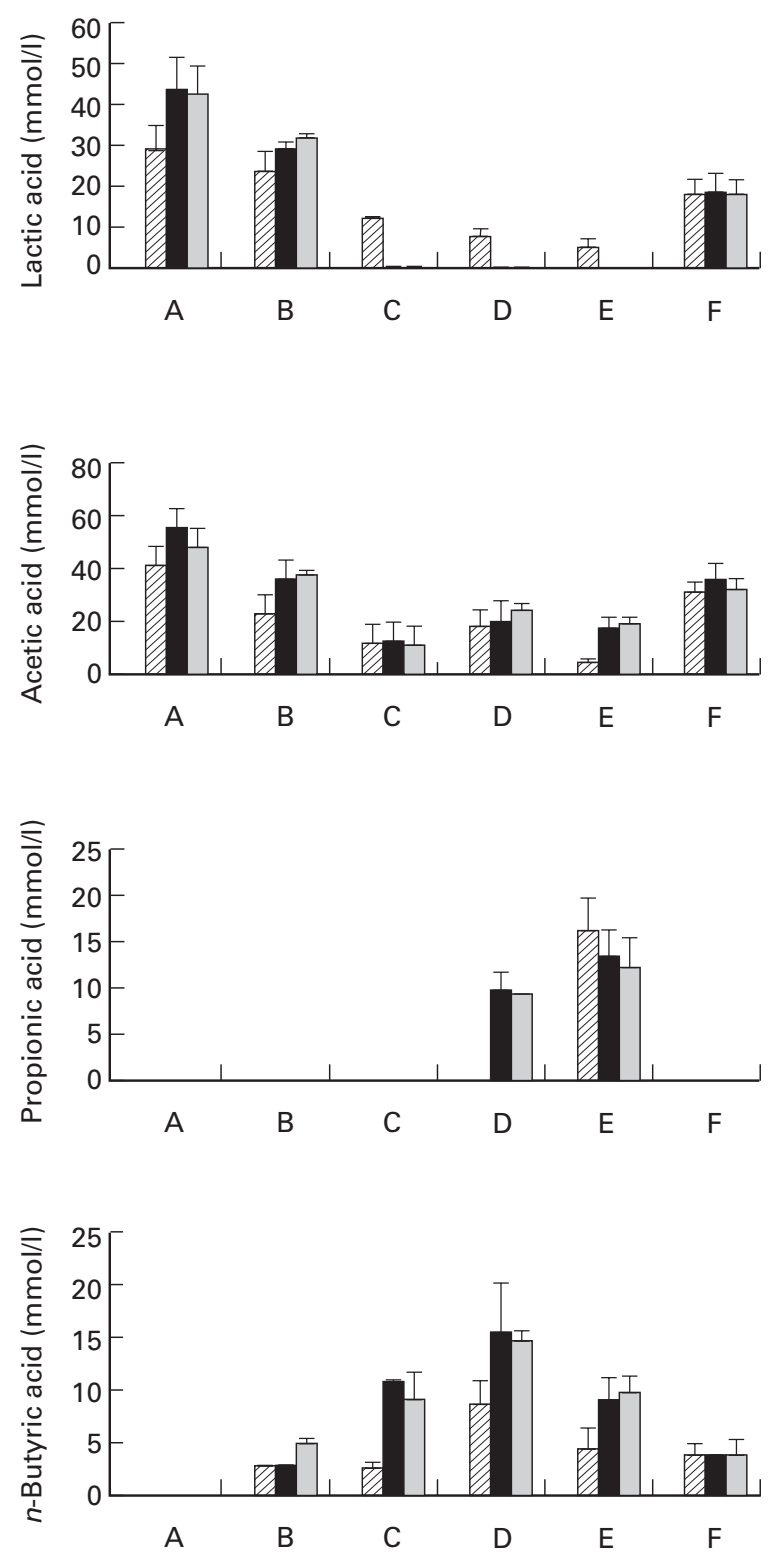

Fig. 3. Production of lactic acid, acetic acid, propionic acid and butyric acid by human gut microflora at $0(\square), 6(\mathbb{Z}), 24$ (ロ) and 48 (畨) $\mathrm{h}$ in batch culture experiments using glucose $(A)$, oligodextran I (B), oligodextran II (C), oligodextran III (D), dextran (E) and fructooligosaccharides $(F)$ as carbohydrate sources. Values are means of duplicate determinations on the same culture, with standard deviations represented by vertical bars.

$5 \mathrm{mmol} / \mathrm{l}$ for oligodextran I. As the average molecular mass of oligodextrans increased, elevated amounts of butyrate were detected, giving a maximum of $14.9 \mathrm{mmol} / \mathrm{l}$ for oligodextran III. Propionate was only formed during the metabolism of dextran and high-molecular-mass oligodextran III (Fig. 3).

\section{Comparative growth of colonic bacteria using a three-stage continuous culture system}

An assessment of bacterial numbers in a validated gut model system (Macfarlane et al. 1998) was carried out, with dextran, oligodextran IV and maltodextrin as carbohydrate sources. Oligodextran IV was chosen because this preparation can currently be manufactured continuously on a larger $(100 \mathrm{~g})$ scale and has a composition similar to that of oligodextran I (Table 1), shown to have a comparable fermentation pattern with FOS in batch cultures. Several groups of intestinal bacteria were selected as markers of the general colonic population to determine how the carbohydrate source may influence the microbial composition.

Steady-state counts of bacterial responses to the carbohydrate sources are presented in Table 2. Anaerobic bacteria were more numerous than aerobes in all three vessels for all the carbohydrate sources tested. Bacteroides species predominated in all three vessels during dextran fermentation but only in V2 and V3 during the oligodextran IV fermentation. Maltodextrin fermentation did not increase Bacteroides species, with steady-state populations being lower than the corresponding ones during oligodextran IV fermentation by $1.9 \log$ and $3.2 \log$ in V1 and V3 respectively. Steady-state clostridia populations in the oligodextran IV fermentation were lower by a minimum of $0.4 \mathrm{log}$ in V2 compared with dextran fermentation. Clostridia counts in maltodextrin fermentation were higher by $1.1 \log$ in $\mathrm{V} 1$ and lower by $1.4 \log$ in V3 compared with oligodextran fermentation.

Oligodextran IV was the best growth substrate for bifidobacteria and lactobacilli from the carbohydrates tested (Table 2) with the highest counts found in V1. Steady-state populations of bifidobacteria and lactobacilli when oligodextran IV was used as the carbohydrate source were higher in all three vessels than the respective populations in the case of dextran and maltodextrin fermentations. Dextran resulted in $1.9 \log$ smaller bifidobacteria and lactobacilli populations in V1 compared with oligodextran IV, whereas maximum differences of $1.4 \log$ and $0.8 \log$ were seen in V3 for bifidobacteria and lactobacilli respectively (Table 2) in the case of maltodextrin.

Total SCFA production in all vessels increased when dextran or oligodextran IV was added into the system, with the highest production recorded in V2 and V3 (Table 3). Lactate was detected only when maltodextrin was the $\mathrm{C}$ source. The production of lactic acid indicates saccharolytic activity, but lactic acid is an electron sink and is normally further oxidized to other SCFA by bacteria growing in the medium. The dextran and oligodextran IV fermentations showed high production of butyric acid, reaching levels of $25.39 \mathrm{mmol} / \mathrm{l}$ and $21.70 \mathrm{mmol} / \mathrm{l}$ in $\mathrm{V} 3$ respectively (Table 3 ).

The slower fermentability of dextran led to a higher persistence of total sugars through V1, allowing $87.5 \%$ of the total carbohydrate source to reach V2 (Fig. 5). The amount of oligodextran IV reaching vessel 2 was $34 \%$, followed by $22 \%$ in the case of maltodextrin.

\section{Discussion}

The aim of the present work was to generate new information on the in vitro utilization of novel oligodextrans produced via membrane reactors (Mountzouris et al. 1999), by human gut microflora, for their possible application as functional food ingredients and in particular as prebiotics. 


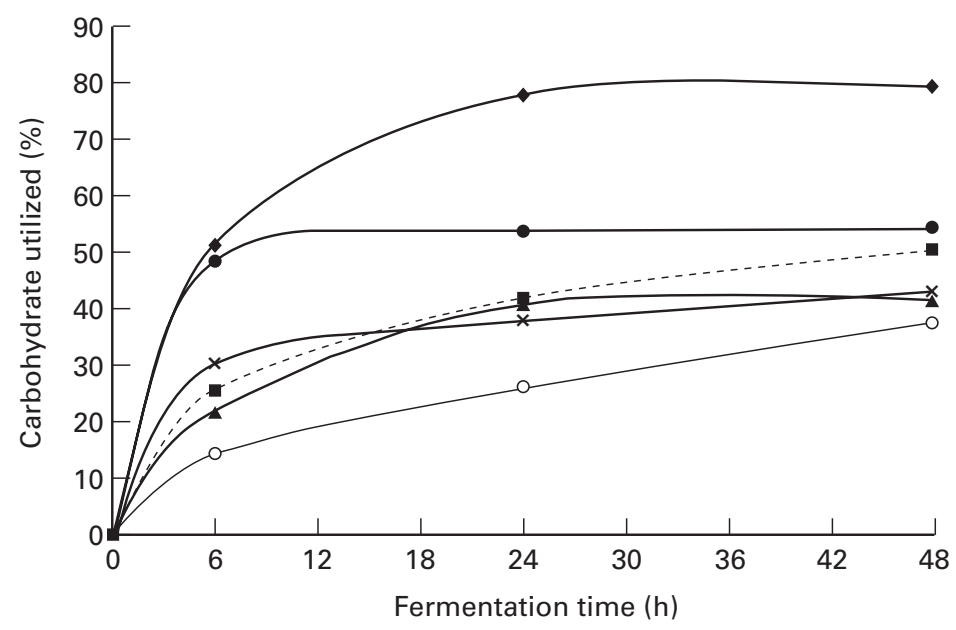

Fig. 4. Carbohydrate utilization (\%) during batch culture fermentations at 0,6 , 24 and $48 \mathrm{~h}$ using different substrates. ( ), Glucose; (•), oligodextran I; $(\times)$, oligodextran II; $(\mathbf{\Lambda})$, oligodextran III; (O), dextran; (- - - -), fructooligosaccharides. Values are means of triplicate determinations on the same culture.

LAB are desirable microbial groups in the gut. Through the dietary route, both probiotics (Fuller, 1989) and prebiotics (Gibson \& Roberfroid, 1995) serve to increase populations of LAB in the large intestine. It would appear that fortification of selected components of the gut flora is nutritionally beneficial. An adjunct to this is the generation of certain fermentation metabolites, of which butyrate has much current significance (Cummings, 1984; Hague et al. 1995; Salminen et al. 1998; Wang \& Friedman, 1998).

The low-molecular-mass oligodextran I (Table 1 and Fig. 1) showed similar bifidogenic behaviour (Fig. 2) to that of FOS in the batch culture study. FOS displayed its

Table 2. Steady-state bacterial counts ( $\log _{10}$ colony-forming units $/ \mathrm{ml}$ ) of selected colonic bacteria in each of the three vessels of a validated three-stage continuous culture system (Macfarlane et al. 1998), using dextran, oligodextran IV and maltodextrin as carbohydrate sources

(Mean values and standard deviations for three samples on the same culture)

\begin{tabular}{|c|c|c|c|c|c|c|}
\hline \multirow[b]{2}{*}{ Bacteria } & \multicolumn{2}{|c|}{ Vessel 1} & \multicolumn{2}{|c|}{ Vessel 2} & \multicolumn{2}{|c|}{ Vessel 3} \\
\hline & Mean & SD & Mean & SD & Mean & SD \\
\hline \multicolumn{7}{|l|}{ Dextran } \\
\hline Coliforms & 6.89 & 0.15 & 6.56 & 0.03 & $5 \cdot 50$ & 0.02 \\
\hline Total aerobes & 7.07 & 0.08 & 6.94 & 0.04 & 6.54 & 0.05 \\
\hline Total anaerobes & $9 \cdot 40$ & 0.04 & 9.93 & 0.07 & 9.17 & 0.04 \\
\hline Bifidobacteria & $7 \cdot 37$ & 0.17 & $7 \cdot 13$ & $0 \cdot 10$ & $7 \cdot 37$ & 0.17 \\
\hline Lactobacilli & $7 \cdot 56$ & $0 \cdot 12$ & 7.42 & $0 \cdot 13$ & 6.98 & 0.03 \\
\hline Bacteroides & 8.87 & 0.13 & 9.94 & 0.03 & 9.61 & 0.02 \\
\hline Clostridia & 8.44 & 0.10 & 9.57 & 0.02 & $9 \cdot 39$ & 0.10 \\
\hline \multicolumn{7}{|l|}{ Oligodextran IV } \\
\hline Čoliforms & 5.68 & 0.01 & 6.41 & 0.06 & 6.84 & 0.14 \\
\hline Total aerobes & 7.47 & 0.03 & 8.05 & 0.01 & 8.23 & 0.06 \\
\hline Total anaerobes & 9.46 & 0.03 & $9 \cdot 61$ & 0.05 & $10 \cdot 13$ & 0.02 \\
\hline Bifidobacteria & $9 \cdot 31$ & 0.12 & 8.67 & 0.07 & 8.44 & 0.06 \\
\hline Lactobacilli & 8.85 & 0.01 & 8.38 & 0.12 & 8.29 & 0.06 \\
\hline Bacteroides & 8.22 & 0.04 & 9.35 & 0.06 & $9 \cdot 10$ & 0.08 \\
\hline Clostridia & $7 \cdot 13$ & 0.07 & $9 \cdot 23$ & 0.08 & 8.84 & 0.06 \\
\hline \multicolumn{7}{|l|}{ Maltodextrin } \\
\hline Coliforms & $6 \cdot 17$ & 0.04 & 6.99 & 0.07 & $6 \cdot 84$ & 0.09 \\
\hline Total aerobes & 6.47 & 0.08 & $7 \cdot 15$ & 0.12 & 6.94 & 0.12 \\
\hline Total anaerobes & 8.44 & 0.05 & 8.68 & 0.05 & $8 \cdot 78$ & 0.02 \\
\hline Bifidobacteria & $8 \cdot 39$ & 0.10 & 7.65 & 0.11 & 6.99 & 0.13 \\
\hline Lactobacilli & 8.38 & 0.07 & 8.11 & 0.10 & 7.46 & 0.11 \\
\hline Bacteroides & $6 \cdot 26$ & $0 \cdot 10$ & 6.02 & 0.04 & $5 \cdot 88$ & 0.06 \\
\hline Clostridia & $8 \cdot 27$ & $0 \cdot 10$ & $7 \cdot 40$ & 0.02 & 7.39 & 0.02 \\
\hline
\end{tabular}


Table 3. Steady-state production of short-chain fatty acids (SCFA) in each of the three vessels of a validated three-stage continuous culture system (Macfarlane et al. 1998), using dextran, oligodextran IV and maltodextrin as carbohydrate sources

(Mean values and standard deviations for two determinations on the same culture)

\begin{tabular}{|c|c|c|c|c|c|c|}
\hline \multirow{2}{*}{$\begin{array}{l}\text { SCFA } \\
(\mathrm{mmol} / \mathrm{l})\end{array}$} & \multicolumn{2}{|c|}{ Vessel 1} & \multicolumn{2}{|c|}{ Vessel 2} & \multicolumn{2}{|c|}{ Vessel 3} \\
\hline & Mean & $\mathrm{SD}$ & Mean & SD & Mean & $\mathrm{SD}$ \\
\hline \multicolumn{7}{|l|}{ Dextran } \\
\hline Lactic acid & 0.00 & - & 0.00 & - & 0.00 & - \\
\hline Acetic acid & 12.51 & 0.29 & 36.63 & 1.47 & 39.04 & 4.8 \\
\hline Propionic acid & $4 \cdot 10$ & 0.08 & 21.59 & 0.11 & $22 \cdot 28$ & 0.88 \\
\hline Butyric acid & $15 \cdot 69$ & 0.50 & $23 \cdot 34$ & $1 \cdot 20$ & $25 \cdot 39$ & $4 \cdot 10$ \\
\hline \multicolumn{7}{|l|}{ Oligodextran IV } \\
\hline Lactic acid & 0.00 & - & 0.00 & - & 0.00 & - \\
\hline Acetic acid & $24 \cdot 14$ & $2 \cdot 72$ & 35.85 & 4.95 & $42 \cdot 72$ & 1.50 \\
\hline Propionic acid & 0.00 & - & $16 \cdot 34$ & 0.69 & 18.63 & 0.32 \\
\hline Butyric acid & 18.00 & 5.03 & $19 \cdot 31$ & 0.92 & $21 \cdot 70$ & 0.78 \\
\hline \multicolumn{7}{|l|}{ Maltodextrin } \\
\hline Lactic acid & 99.60 & 7.65 & 94.47 & 0.97 & 12.94 & $2 \cdot 26$ \\
\hline Acetic acid & $7 \cdot 88$ & 1.78 & $13 \cdot 71$ & 0.52 & 31.58 & 2.55 \\
\hline Propionic acid & 0.00 & - & 0.00 & - & 46.69 & 1.64 \\
\hline Butyric acid & 1.37 & 1.94 & 3.71 & 0.50 & 12.64 & 0.71 \\
\hline
\end{tabular}

well-known bifidogenic character (Wang \& Gibson, 1993; Gibson \& Wang, 1994) by stimulating the proliferation of bifidobacteria during the first $6 \mathrm{~h}$ of batch culture (Fig. 2), whilst decreasing the counts of other less desirable bacteria (i.e. bacteroides, clostridia). The bifidogenic properties of the low-molecular-mass oligodextran IV (Table 1) were also seen during its fermentation in the more representative three-stage continuous culture system. Bifidobacteria and lactobacilli grew at high numbers $(8 \cdot 3-9.3 \mathrm{log})$ in all the vessels and their steady-state populations were higher by far (i.e. at least $0.5 \mathrm{log}$ for the lactobacilli and $0.9 \mathrm{log}$ for the bifidobacteria) than the respective ones in the case of dextran and maltodextrin fermentations (Table 2). Overall, the LAB populations seen in the oligodextran IV fermentation reported here were higher (i.e. at least $2 \cdot 1 \log$ for lactobacilli and $0.5 \mathrm{log}$ for bifidobacteria) than the respective steady-state LAB populations of FOS fermentation in a study conducted by Gibson \& Wang (1994), using a similar three-stage continuous culture system.

In the batch cultures, the low-molecular-mass oligodextran I resulted in higher amounts of bifidobacteria during the early stages of the fermentation compared with dextran and oligodextrans II and III (Fig. 1). In the continuous culture system, dextran steady-state LAB populations were also at least $1 \log$ lower in all the vessels than the respective ones from the low-molecular-mass oligodextran IV (Table 2). The bifidogenic differences between the high-molecularmass oligodextrans II, III and dextran and the low-molecularmass oligodextrans (I and IV) could possibly be attributed to the differences in their DP. The effect of DP of the $\beta-2 \rightarrow 1$ fructans on the inulinase activities has been considered as the reason why FOS has been shown to be the preferred growth substrate by many strains of bifidobacteria compared with glucose, inulin and branched FOS (Gibson \& Wang,

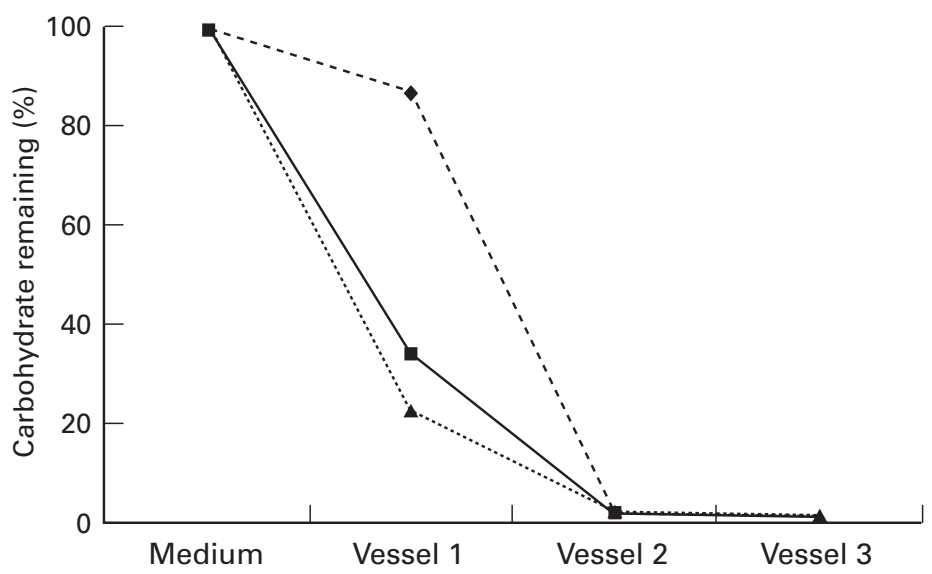

Fig. 5. Percentage of carbohydrate remaining in each vessel of the threestage continuous culture system at steady-state, using different substrates. $(-\rightarrow-)$, Dextran; $(-\mathbf{-})$, oligodextran IV; $(\cdots \mathbf{\Delta} \cdots)$, maltodextrin. Values are means of triplicate determinations on the same culture. 
1994). In the case of dextranases the extraction of exo- and endo-dextranases from Bifidobacterium bifidum has been reported (Sidebotham, 1974). However, the effect of oligodextran DP on the dextranase activities of various colonic genera is unknown and will have to be determined in future studies.

The carbohydrate utilization pattern of the oligodextrans and dextran in the batch cultures was shown to be dependent on their overall molecular mass, with oligodextran I being utilized to a greater extent than dextran (Fig. 4). Similar behaviour was also observed in the three-stage continuous system where dextran persisted through V2 to a greater extent than oligodextran IV (Fig. 5). In vivo, this might suggest that higher molecular-mass oligodextrans are more likely to persist through the colon.

In the batch cultures it was seen that clostridia counts were reduced after $6 \mathrm{~h}$ but also persisted up to $24 \mathrm{~h}$ (Fig. 2) in the cases of oligodextrans II, III and dextran. Use of a validated gut model showed that bacterial counts during dextran and oligodextran IV fermentation gave elevated numbers of clostridia as the $\mathrm{pH}$ increased. Clostridia are known not to grow in acidic conditions (Gibson \& Roberfroid, 1995), with Clostridium perfringens not growing at culture pH values below 5 (Wang \& Gibson, 1993). Clostridium species were promoted mainly in V2 in dextran and oligodextran IV fermentation, where the $\mathrm{pH}$ was higher (i.e. $\mathrm{pH}$ 6.2) than in V1 and carbohydrate was still overflowing in high amounts from V1 (Fig. 5). Maltodextrin fermentation was characterized by high numbers of clostridia in V1 which were subsequently reduced by $0.8 \log$ in $\mathrm{V} 2$ and V3 (Table 2).

It is clear that gut bacteria cannot be reliably identified through phenotypic procedures (Collins \& Gibson, 1999). Hence, the in vitro study reported here used a molecular characterization of some predominant fermentative organisms.

Sequencing of the 16S-rRNA genes of random isolates from a growth medium designed to be selective for Clostridium species showed that the predominant species recovered was Clostridium perfringens. This observation was in good agreement with the results reported by Kohomoto et al. (1991) in which increases in the counts of clostridia and especially $C$. perfringens in the faeces of healthy adults were observed following oral administration of commercial IMO. Previously the growth of $C$. perfringens on commercial IMO had been shown by Kohmoto et al. (1988).

Butyrate is not a major end-fermentation product of bacteroides, or of the common target probiotic bacteria (i.e. bifidobacteria and lactobacilli), whereas it is for eubacteria and clostridia. Interestingly, butyrate generation in V2 and V3, which correspond to the transverse and distal colons respectively, was enhanced with both the dextran and oligodextran IV (Table 3), indicating a saccharolytic metabolism. As such, clostridia may well have been contributing towards butyrate formation.

More importantly dextran and oligodextran resulted in the production of almost twice the amount of butyrate compared with maltodextrin (Table 3). Butyrate currently attracts more attention than other SCFA as a desirable metabolite of gut bacterial function, through its interaction with colonocytes (Cummings, 1984). Butyrate is the primary respiratory fuel in the distal colonic mucosa. The epithelial cells of the intestinal surface obtain from its metabolism $60-70 \%$ of the energy needed (Scheppach, 1994). Anti-neoplastic properties have also been attributed to butyrate: in cancer-cell studies it prolongs doubling time and reduces the growth rate of human large-bowel cancer cells (Cummings \& Macfarlane, 1991; Gamet et al. 1992; Scheppach et al. 1995). Butyrate has been shown in vitro not only to induce apoptosis in a number of colonic tumour cell lines but also to be the most effective inducer of apoptosis compared with propionate and acetate (Hague et al. 1995). SCFA mixtures that mimic the digestion products of wheat bran, oat bran and pectin have been shown to induce growth arrest in a human colon carcinoma cell line by induction of the p21 and p27 cyclin-directed kinase inhibitors (Wang \& Friedman, 1998). The relative efficiency of these SCFA mixtures in inducing growth arrest was directly proportional to the amount of butyrate present in the mixture (Wang \& Friedman, 1998). The data obtained from the gut model system during the oligodextran IV fermentation (Table 3) showed a production of $42.7 \mathrm{mmol} / \mathrm{l}$ acetate, $18.7 \mathrm{mmol} / 1$ propionate and $21.70 \mathrm{mmol} / \mathrm{l}$ butyrate in $\mathrm{V} 3$ of the gut model which represents the left colon, the predominant site for onset of colon cancer. In developed countries the colon is the second commonest site of cancer, and this disease is a major source of sickness and mortality. The possible biological properties of butyrate directed towards bowel cancer are of relevance, and it would therefore seem to be desirable to promote its production in the colon.

A dietary component that allows both increased prevalence of LAB, as well as increased butyrate levels would appear to hold much promise as a functional food ingredient. In the present study, it has been observed that the novel low-molecular-mass oligodextran IV promoted the growth of bifidobacteria and lactobacilli while producing butyrate.

The in vitro studies described in this paper suggest that oligodextrans have potential as modulatory agents for the gut microflora. The next phase of this research is to scale up production in order to facilitate in vivo studies.

\section{Acknowledgement}

The authors would like to thank the European Commission FAIR-CT 96-5022 for funding part of this project.

\section{References}

Collins MD \& Gibson GR (1999) Probiotics, prebiotics and symbiotics: approaches for modulating the microbial ecology of the gut. American Journal of Clinical Nutrition 69, S1052S1057.

Cummings JH (1984) Microbial digestion of complex carbohydrates in man. Proceedings of the Nutrition Society 43, 35-44.

Cummings JH \& Macfarlane GT (1991) The control and consequences of bacterial fermentation in the human colon. Journal of Applied Bacteriology 70, 443-459.

Delzenne NM \& Roberfroid MR (1994) Physiological effects of non-digestible oligosaccharides. Lebensmittel-Wissenschaft und Technologie 27, 1-6.

Dubois M, Gilles KA, Hamilton JK, Rebers PA \& Smith F (1956) Colorimetric method for determination of sugars and related substances. Analytical Chemistry 28, 350-356. 
Fuller R (1989) Probiotics in man and animals. Journal of Applied Bacteriology 66, 365-378.

Gamet L, Daviaud D, Denis-Pouxviel C, Remesy C \& Murat JC (1992) Effects of short-chain fatty acids on growth and differentiation of human colon-cancer cell line HT29. International Journal of Cancer 52, 286-289.

Gibson GR \& Roberfroid MB (1995) Dietary modulation of the human colonic microbiota: introducing the concept of prebiotics. Journal of Nutrition 125, 1401-1412.

Gibson GR \& Wang X (1994) Bifidogenic properties of different types of fructo-oligosaccharides. Food Microbiology 11, 491-498.

Hague A, Elder DJE, Hicks DJ \& Paraskeva C (1995) Apoptosis in colorectal tumour cells: induction by the short chain fatty acids butyrate, propionate and acetate and by the bile salt deoxycholate. International Journal of Cancer 60, 400-406.

Japanscan (1998) Functional Foods and Drinks in Japan. Japanscan Food Industry Bulletin. Leatherhead: Leatherhead Food Research Association.

Kaneko T, Yokoyama A \& Suzuki M (1995) Digestibility characteristics of isomaltooligosaccharides in comparison with several saccharides using the rat jejunum loop method. Bioscience Biotechnology and Biochemistry 59, 1190-1194.

Kohmoto T, Fukui F, Takaku H, Machida Y, Arai M \& Mitsuoka T (1988) Effect of isomalto-oligosaccharide on human fecal flora. Bifidobacteria Microflora 7, 61-69.

Kohmoto T, Fukui F, Takaku H \& Mitsuoka T (1991) Doseresponse test of isomaltooligosaccharides for increasing fecal bifidobacteria. Agricultural and Biological Chemistry 55, 2157 2159.

McCartney AL \& Gibson GR (1998) The application of prebiotics in human health and nutrition. In Proceedings Lactic 97. Which Strains? For Which Products?, pp. 59-73. Normandie: Adria.

Macfarlane GT, Gibson GR \& Cummings JH (1992) Comparison of fermentation reactions in different regions of the human colon. Journal of Applied Bacteriology 72, 57-64.
Macfarlane GT, Macfarlane S \& Gibson GR (1998) Validation of a three-stage compound continuous culture system for investigating the effect of retention time on the ecology and metabolism of bacteria in the human colonic microbiota. Microbial Ecology 35, 180-187.

Mizota T (1996) Functional and nutritional foods containing bifidogenic factors. Bulletin of the International Dairy Federation 313, 31-35.

Mountzouris KC, Gilmour SG, Grandison AS \& Rastall RA (1999) Modeling of oligodextran production in an ultrafiltration stirredcell membrane reactor. Enzyme Microbial Technology 24, 7585.

Salminen S, Bouley C, Boutron-Ruault MC, Cummings JH, Franck A, Gibson GR, Isolauri E, Moreau MC, Roberfroid M \& Rowland IR (1998) Functional food science and gastrointestinal physiology and function. British Journal of Nutrition $\mathbf{8 0}$, S147-S171.

Scheppach W (1994) Effects of short chain fatty acids on gut morphology and function. Gut 35, Suppl. 1, S35-S38.

Scheppach W, Bartram HP \& Richter F (1995) Role of short chain fatty acids in the prevention of colorectal cancer. European Journal of Cancer 31A, 1077-1080.

Sidebotham RL (1974) Dextrans. In Advances in Carbohydrate Chemistry and Biochemistry, vol. 30, pp. 371-444 [RS Tipson and D Horton, editors]. New York, NY: Academic Press.

Tomomatsu H (1994) Health effects of oligosaccharides. Food Technology October issue, 61-65.

Wang J \& Friedman EA (1998) Short-chain fatty acids induce cell cycle inhibitors in colonocytes. Gastroenterology 114, 940946.

Wang X \& Gibson GR (1993) Effects of the in vitro fermentation of oligofructose and inulin by bacteria growing in the human large intestine. Journal of Applied Bacteriology 75, 373-380.

Yaeshima T (1996) Benefits of bifidobacteria to human health. Bulletin of the International Dairy Federation 313, 36-42. 